\title{
Parametric oscillator in a Kerr medium: evolution of coherent states
}

\author{
R. Román-Ancheyta, ${ }^{1, *}$ M. Berrondo, ${ }^{2}$ and J. Récamier ${ }^{1}$ \\ ${ }^{1}$ Instituto de Ciencias Fisicas, Universidad Nacional Autónoma de México, Apartado Postal 48-3, Cuernavaca, Morelos 62251, Mexico \\ ${ }^{2}$ Department of Physics and Astronomy, Brigham Young University, Provo, Utah 84602, USA \\ *Corresponding author: ancheyta6@gmail.com
}

Received 13 April 2015; revised 16 June 2015; accepted 21 June 2015; posted 25 June 2015 (Doc. ID 237908); published 17 July 2015

\begin{abstract}
We study the temporal evolution of a coherent state under the action of a parametric oscillator and a nonlinear Kerr-like medium. We make use of the interaction-picture representation and use an exact time-evolution operator for the time-independent part of the Hamiltonian. We approximate the interaction-picture Hamiltonian in such a way as to make it a member of a Lie algebra. The corresponding time-evolution operator behaves like a squeezing operator due to the temporal dependence of the oscillator's frequency. We analyze the probability amplitude, the auto-correlation function, and the Husimi distribution function for different Hamiltonian parameters. We find very good agreement between our approximate results and converged numerical calculations. (๑) 2015 Optical Society of America
\end{abstract}

OCIS codes: (270.5290) Photon statistics; (270.6570) Squeezed states.

http://dx.doi.org/10.1364/JOSAB.32.001651

\section{INTRODUCTION}

Coherent states were introduced by Schrödinger in 1926 in the early stages of quantum mechanics [1]. These quantum states are characterized by the fact that the trajectory of the center of the coherent wave packet evolves in time in the same way as a classical harmonic oscillator and its dispersion takes the minimum value allowed by Heisenberg's principle. These facts make them especially useful as a link between the classical and the quantum theories.

Much later, in 1963, Glauber introduced the field-coherent states, which are coherent states for the electromagnetic field. These states play an important role in quantum optics [2,3]. The development of lasers made it possible to prepare light fields that are very close to the coherent states. Glauber showed that the field-coherent states can be obtained from any one of the three mathematical definitions: (i) as the right-hand eigenstates of the boson annihilation operator $\hat{a}|\alpha\rangle=\alpha|\alpha\rangle$ with $\alpha$ a complex number, (ii) as those states obtained by application of the displacement operator upon the vacuum state of the harmonic oscillator $D(\alpha)|0\rangle=|\alpha\rangle$ with $D(\alpha)=\exp \left(\alpha \hat{a}^{\dagger}-\alpha^{*} \hat{a}\right)$, and (iii) as the quantum states with a minimum uncertainty product $(\Delta p)(\Delta q)=\hbar / 2$ with $\Delta q=\Delta p$. The coherent states obtained from any one of these definitions are identical when one makes use of harmonic oscillator algebra. Subject to a linear interaction, a coherent state evolves into a new coherent state; that is, they show temporal stability [ㄴ,,$\underline{5}$. Nieto and Simmons [ $\underline{6}-8]$ generalized the notion of coherent states for potentials different from the harmonic oscillator with unequally spaced energy levels such as the Morse potential and the PöschlTeller potential. The resulting states are localized, follow the classical motion, and disperse as little as possible in time. Gazeau and Klauder [9] proposed a generalization for systems with one degree of freedom possessing discrete as well as continuous spectra. These states present continuity of labeling, a resolution of the identity, and temporal stability. Man'ko et al. [10] introduced coherent states of an $f$-deformed algebra as eigenstates of a deformed annihilation operator $\hat{A}=\hat{a} f(\hat{n})$ where $f(\hat{n})$ is a function of the number operator that specifies the deformation. These states present nonclassical properties like squeezing and antibunching. The properties of their even and odd combinations have also been studied $[11,12]$.

In the presence of a nonlinear interaction, field-coherent states evolve into nonclassical states. This can be achieved experimentally by passing a coherent state through a Kerr medium resulting in the appearance of distinguishable macroscopic superpositions of coherent states, which are the so-called cat states $[13,14]$.

The parametric harmonic oscillator, namely, a harmonic oscillator with a time-dependent frequency, has been studied from several points of view: using the method of adiabatic invariants [15-19], super symmetric quantum mechanics [20], algebraic methods $[\underline{21}, \underline{22}]$, and different approximation methods [23]. A particularly relevant realization of the parametric oscillator is cavity quantum electrodynamics where the frequency of a given field mode in the cavity can change in time due to the motion of the cavity walls or to changes in the 
dielectric function of the medium [24]. For instance, Wineland et al. [25] theoretically and experimentally analyzed both the loss of coherence caused by fluctuations in the trap parameters and in the amplitude and frequency of the laser beams, heating due to collisions with background gas, internal state decoherence due to radiative decay, and coupling to spectator levels.

In this work we consider a nonlinear system corresponding to a single mode field propagating in a Kerr-like medium immersed in a cavity with a time-dependent frequency. In Section 2 we write the Hamiltonian and construct its timeevolution operator. In Section 3 we follow the evolution of coherent states under the nonlinear Hamiltonian and analyze some of their statistical properties like probability distributions, autocorrelation function, and Husimi function. Finally, in Section $\underline{4}$ we present our conclusions.

\section{THEORY}

Consider a parametric harmonic oscillator immersed in a Kerr-like medium. Its Hamiltonian is given by [26]

$$
\hat{H}(t)=\frac{1}{2}\left[\hat{p}^{2}+\Omega^{2}(t) \hat{q}^{2}\right]+\hat{H}_{\mathrm{Kerr}},
$$

where $\Omega(t)$ is an explicit time-dependent frequency and $\hat{H}_{\mathrm{Kerr}}$ has to do with the Kerr-like medium. We can define the usual annihilation, creation, and number operators as

$$
\begin{aligned}
& \hat{a}=\frac{1}{\sqrt{2 \Omega_{0}}}\left(\Omega_{0} \hat{q}+i \hat{p}\right), \quad \hat{a}^{\dagger}=\frac{1}{\sqrt{2 \Omega_{0}}}\left(\Omega_{0} \hat{q}-i \hat{p}\right), \\
& \hat{n}=\hat{a}^{\dagger} \hat{a},
\end{aligned}
$$

where we have set $\hbar=1$ and we write the Kerr medium [27] as $\hat{H}_{\text {Kerr }}=\chi \hat{n}^{2}$, with $\chi$ a constant proportional to a third-order nonlinear susceptibility $\chi^{(3)}$, which is, in general, a small number [28]. To be specific, in what follows we will choose $\Omega(t)=$ $\Omega_{0}\left[1+2 \kappa \cos \left(2 \Omega_{0} t\right)\right]$ [29] with $\kappa$ also a small parameter. The Hamiltonian can be written in terms of $\hat{a}^{\dagger}, \hat{a}$, and $\hat{n}$ as

$$
\hat{H}(t)=\Omega_{0}(\hat{n}+1 / 2)+\chi \hat{n}^{2}+g(t)\left(\hat{a}^{2}+\hat{a}^{\dagger 2}+2 \hat{n}+1\right),
$$

and $g(t)=\Omega_{0} \kappa \cos \left(2 \Omega_{0} t\right)\left(1+\kappa \cos \left(2 \Omega_{0} t\right)\right)$. The timeevolution operator corresponding to the nonlinear timeindependent part of the Hamiltonian is given by

$$
\hat{U}_{0}=\exp \left(-i \Omega_{0} t(\hat{n}+1 / 2)-i t \chi \hat{n}^{2}\right),
$$

and we can write the time-dependent Hamiltonian in the interaction picture as

$$
\hat{H}_{I}(t)=g(t)\left(e^{-2 i \Omega(\hat{n}) t} \hat{a}^{2}+\hat{a}^{\dagger 2} e^{2 i \Omega(\hat{n}) t}+2 \hat{n}+1\right),
$$

where we have used the fact that $f(\hat{n}) \hat{a}=\hat{a} f(\hat{n}-1)$, $f(\hat{n}) \hat{a}^{\dagger}=\hat{a}^{\dagger} f(\hat{n}+1)$, and the effective frequency $\Omega(\hat{n})=$ $\Omega_{0}+2 \chi(1+\hat{n})$ are functions of the number operator.

Notice that the time-evolution operator $\hat{U}_{0}$ is exact and includes anharmonicity due to the Kerr medium explicitly. The interaction-picture Hamiltonian given in Eq. (5) is also exact. The operators given in the interaction-picture Hamiltonian do close under commutation, however they have an explicit time dependence and the Wei-Norman theorem cannot be applied. Nevertheless, the set $\left\{\hat{a}^{\dagger 2}, \hat{n}+1 / 2, \hat{a}^{2}\right\}$ also forms the basis of a
Lie algebra [the su( 1,1$)$ algebra] closed under commutation. In order to attain a more manageable Hamiltonian that can be written as a linear combination of time-independent operators, we approximate the exponentials by their average value [30]; that is, we make the replacement $\exp [ \pm 2 i \Omega(\hat{n}) t]$ by $\left\langle\alpha_{0}|\exp [ \pm 2 i \Omega(\hat{n}) t]| \alpha_{0}\right\rangle$ obtaining the approximate interactionpicture Hamiltonian:

$$
\begin{aligned}
\tilde{H}_{I}(t)= & g(t)\left(e^{-2 i\left(\Omega_{0}+2 \chi\right) t} \hat{a}^{2}\left\langle e^{-4 i \chi t \hat{n}}\right\rangle\right. \\
& \left.+\hat{a}^{\dagger 2} e^{2 i\left(\Omega_{0}+2 \chi\right) t}\left\langle e^{4 i \chi t \hat{n}}\right\rangle+2 \hat{n}+1\right),
\end{aligned}
$$

where the expectation value is taken with respect to an initial coherent state. The resulting approximate Hamiltonian is similar to that of a degenerate parametric amplifier [27], where a nonlinear medium is pumped by a strong laser inducing the emission and absorption of photon pairs [31].

With this simplification, $\tilde{H}_{I}(t)$ is an element of the Lie algebra with time-dependent coefficients, and the corresponding time-evolution operator may be written exactly in the product form $[\underline{32}, \underline{33}]$

$$
\tilde{H}_{I}(t)=\sum_{n=1}^{4} f_{n}(t) \hat{X}_{n}, \quad \hat{U}_{I}(t)=\prod_{n=1}^{4} e^{\gamma_{n}(t) \hat{X}_{n}},
$$

with initial conditions $\gamma_{n}\left(t_{0}\right)=0$. We have chosen the ordering $\hat{X}_{1}=\hat{a}^{\dagger 2}, \hat{X}_{2}=\hat{n}, \hat{X}_{3}=\hat{a}^{2}$, and $\hat{X}_{4}=1$. The average takes the form $\left\langle\alpha_{0}\left|e^{ \pm 4 i \chi t \hat{n}}\right| \alpha_{0}\right\rangle=\exp \left[\left|\alpha_{0}\right|^{2}\left(e^{ \pm 4 i \chi t}-1\right)\right]$. The complex, time-dependent functions $\gamma_{n}(t)$ needed to construct $\hat{U}_{I}(t)$ are obtained from the following set of coupled, nonlinear, ordinary differential equations obtained after substitution of Eq. (7) in Schrödinger's equation,

$$
\begin{gathered}
\dot{\gamma}_{1}=-i\left(f_{1}+2 \gamma_{1} f_{2}+4 \gamma_{1}^{2} f_{3}\right), \\
\dot{\gamma}_{2}=-i\left(f_{2}+4 \gamma_{1} f_{3}\right), \\
\dot{\gamma}_{3}=-i f_{3} e^{2 \gamma_{2}}, \\
\dot{\gamma}_{4}=-i\left(f_{4}+2 \gamma_{1} f_{3}\right),
\end{gathered}
$$

where the dot means the time derivative. These equations can be solved either analytically or numerically. The equation for $\gamma_{1}(t)$, being a Riccati equation and the equations for the other $\gamma^{\prime} s(t)$, can be obtained by integration.

\section{STATISTICAL PROPERTIES}

\section{A. Probability Distributions}

Once we have the explicit form for the time-evolution operator, we can evaluate the temporal evolution of a coherent state $|\alpha\rangle$ by means of

$$
|\alpha ; t\rangle=\hat{U}_{0}(t) \hat{U}_{I}(t)|\alpha\rangle,
$$

which is given explicitly as

$$
|\alpha ; t\rangle=N_{\alpha} \sum_{l, m}^{\infty} \frac{\left(\alpha e^{\gamma_{2}-i \Omega_{0} t}\right)^{l}\left(\gamma_{1} e^{-2 i \Omega_{0} t}\right)^{m}}{m ! l ![(l+2 m) !]^{-1 / 2}} e^{-i \chi t(l+2 m)^{2}}|l+2 m\rangle,
$$

with $N_{\alpha}=\exp \left(-i \Omega_{0} t / 2+\gamma_{4}+\alpha^{2} \gamma_{3}-|\alpha|^{2} / 2\right)$.

The probability of finding the $k^{\prime}$ th excited state in the distribution at time $t$ is given by $P_{k}(\alpha ; t)=|\langle k \mid \alpha ; t\rangle|^{2}$. We obtain 


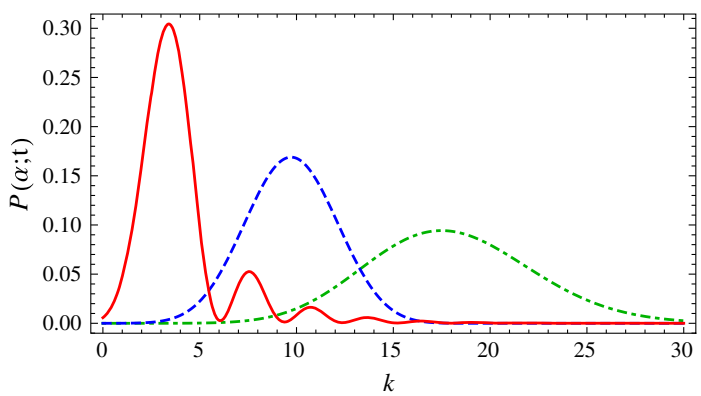

Fig. 1. Probability distribution for the evolved coherent state with Hamiltonian parameters. $\chi=0, \kappa=0.05, \alpha=3+i 3, \alpha_{0}=\sqrt{18}$ at times $t=0$ (green), $t=2 \pi$ (blue), and $t=6 \pi$ (red).

$$
P_{k}(\alpha ; t)=\left|N_{\alpha} \sqrt{k !} \sum_{m=0}^{[k / 2]} \frac{\gamma_{1}^{m}\left(\alpha e^{\gamma / 2}\right)^{k-2 m}}{m !(k-2 m) !}\right|^{2},
$$

where $[\theta]$ means the integer part of $\theta$.

In Fig. $\underline{1}$ we show the probability distribution as a function of $k$ for three different values of time for the case when the Kerr term $\chi=0$ corresponding to a parametric harmonic oscillator. Here the evolution due to the time-independent part of the Hamiltonian is that of a harmonic oscillator and the interaction-picture Hamiltonian is a linear combination of the operators $\left\{\hat{a}^{2}, \hat{a}^{\dagger 2}, \hat{n}\right\}$ so that its time-evolution operator is similar to a squeezing operator $S=\exp \left[\frac{1}{2}\left(\zeta^{*} \hat{a}^{2}-\zeta \hat{a}^{\dagger 2}\right)\right]$. The Hamiltonian parameters used in this example are $\kappa=0.05$, $\alpha=3+i 3$, and times $t=0$ (green), $t=2 \pi$ (blue), and $t=$ $6 \pi$ (red). At the initial time the probability distribution is a Poissonian centered at $\langle n\rangle=18$ as corresponds to a usual coherent state. At $t=2 \pi$ its width has decreased and is now centered at $k=10$. Finally at $t=6 \pi$ its maximum is located at $k=3$. Its width is even smaller and it presents noticeable oscillations after an initial bell shape. These oscillations are evidence of the nonclassicality of the state and are due to the quantum interferences in phase space.

\section{B. Auto-Correlation Function}

The auto-correlation function is defined as the overlap [34]

$$
F(t)=\langle\Psi(0) \mid \Psi(t)\rangle,
$$

and it takes large values at times whenever the wave packet resembles the original one. When the overlap is complete, we have a complete revival. Otherwise, we may have fractional revivals when the overlap is a fraction $(1 / q)$ of the total probability. The phenomenon of wave-packet revivals (complete or fractional) has been observed in many experimental situations in atomic and molecular systems $[\underline{35}, \underline{36}]$.

Using the explicit forms of the time-evolution operators $\hat{U}_{0}$ and $\hat{U}_{I}$, the time-dependent coherent state $|z ; t\rangle$ can be expanded in terms of the number eigenkets $|n\rangle$ as given by Eq. (10). The corresponding auto-correlation function is

$$
\begin{aligned}
F(t)= & e^{-i \Omega_{0} t / 2+\gamma_{4}+z^{2} \gamma_{3}-|z|^{2}} \sum_{k, l,=0}^{\infty} e^{-i \chi t(k+2 l)^{2}} \\
& \times\left(|z|^{2} e^{\gamma_{2}-i \Omega_{0} t}\right)^{k}\left(z^{* 2} \gamma_{1} e^{-i 2 \Omega_{0} t}\right)^{l} /(k ! l !) .
\end{aligned}
$$

When the coefficient of the nonlinear term $\chi$ vanishes, we deal with a parametric oscillator (Fig. $\underline{2}$ top) and the

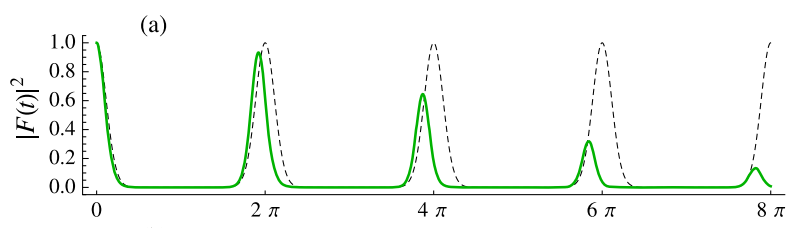

(b)

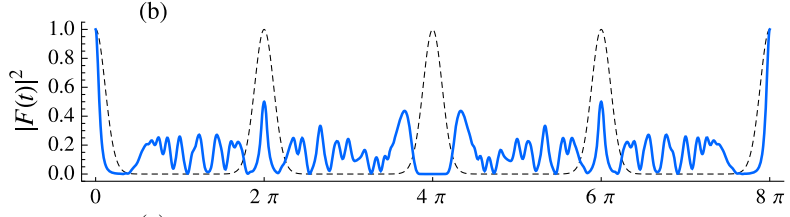

(c)

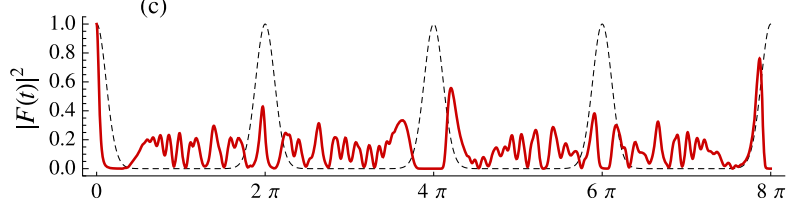

Fig. 2. Absolute value squared of the auto-correlation function $|F(t)|^{2}$ as a function of time for a state $|z ; t\rangle$. Hamiltonian parameters, (a) $\kappa=0.05, \chi=0$; (b) $\kappa=0, \chi=0.25$; (c) $\kappa=0.25, \chi=0.25$. The black dotted line is for a field-coherent state $\left|z e^{i \Omega_{0} t}\right\rangle$. In all cases we have $z=2, \alpha_{0}=2$, and $\Omega_{0}=1$.

auto-correlation function for the coherent state $|z ; t\rangle$ is a periodic decreasing function of time. Its explicit form can be written as an exponential:

$$
\begin{aligned}
F(t)_{\chi=0}= & \exp \left(-i \Omega_{0} t / 2+\gamma_{4}+z^{2} \gamma_{3}-|z|^{2}\right. \\
& \left.+z^{* 2} \gamma_{1} e^{-i 2 \Omega_{0} t}+|z|^{2} e^{\gamma_{2}-i \Omega_{0} t}\right) .
\end{aligned}
$$

As a reference we show in black the temporal evolution for a field-coherent state $\left|z e^{i \Omega_{0} t}\right\rangle$.

When the coefficient corresponding to the temporal dependence of the frequency $\kappa \ll 1$ and that of the nonlinear term is non-negligible (and we thus have a nonlinear oscillator), we show in Fig. $\underline{2}$ (intermediate) the auto-correlation function for a coherent state $|z ; t\rangle$. We can see that there are periodic fractional and complete revivals with the revival time $T_{\text {rev }}=4 \pi \hbar /\left|E^{\prime \prime}\left(n_{0}\right)\right|=8 \pi$. Notice also that the autocorrelation function is symmetric with respect to $T_{\text {rev }} / 2=4 \pi$. In Fig. 2 (bottom) we show the case when neither $\kappa$ nor $\chi$ are negligible. We have a parametric nonlinear oscillator. Here the auto-correlation function shows only fractional revivals. The revival near $8 \pi$ is not complete and appears at a time slightly earlier than $T_{\text {rev }}$. Notice also that the periodicity with respect to $t=4 \pi$ has been lost.

In Fig. $\underline{3}$ we plot the absolute value squared of the autocorrelation function $|F(t)|^{2}$ as a function of time for a state $|z ; t\rangle$ with Hamiltonian parameters $\kappa=0.25, \chi=0.25$. In red we present the result obtained using the time-evolution operator $\hat{U}_{I}(t)$ obtained from the approximate interaction-picture Hamiltonian $\tilde{H}_{I}(t)$. In black we present the result obtained when the evolution of the system is done numerically taking into account the full Hamiltonian given by Eq. (5). Notice the almost perfect agreement between the converged numerical result and our approximate result. This is an indication of the quality of our approximation when dealing with the interaction-picture Hamiltonian. We stress the fact that the 


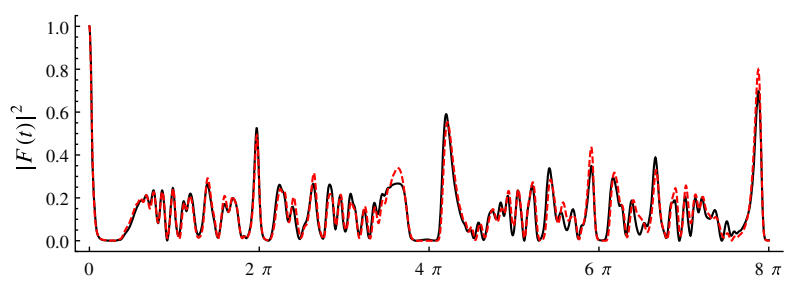

Fig. 3. Absolute value squared of the auto-correlation function $|F(t)|^{2}$ as a function of time for a state $|z ; t\rangle$. Converged numerical results in black and our approximate results in red. Hamiltonian parameters $\kappa=0.25, \chi=0.25$. In all cases we have $z=2$, $\alpha_{0}=2$, and $\Omega_{0}=1$.

nonlinearity $\chi \hat{n}^{2}$ has been taken into account exactly by means of the time-evolution operator $\hat{U}_{0}$.

\section{Husimi-Q Distribution}

The Husimi function is defined as the expectation value of the density operator with respect to the coherent state, and it plays the role of a phase-space probability distribution. It is given as

$$
Q(\alpha)=\frac{1}{\pi}\langle\alpha|\hat{\rho}| \alpha\rangle,
$$

and if $\hat{\rho}(t)=|z ; t\rangle\langle z ; t|$ we get

$$
Q^{|z ; t\rangle\langle z ; t|}(\alpha)=\frac{1}{\pi}\langle\alpha \mid z ; t\rangle\langle z ; t \mid \alpha\rangle=\frac{1}{\pi}|\langle\alpha \mid z ; t\rangle|^{2},
$$

with the normalization condition

$$
\int Q(\alpha) \mathrm{d}^{2} \alpha=1
$$

The $Q$ function is always positive. Taking the explicit form obtained for the time-dependent coherent state $|z ; t\rangle$ and projecting upon a coherent state $|\alpha\rangle$, we get the overlap

$$
\begin{aligned}
\langle\alpha \mid z ; t\rangle= & \sum_{l, m,=0}^{\infty} \frac{\left(z \alpha^{*} e^{\gamma_{2}-i \Omega_{0} t}\right)^{l}}{l !} \frac{\left(\alpha^{* 2} \gamma_{1} e^{-i 2 \Omega_{0} t}\right)^{m}}{m !} \\
& \times N_{z} e^{-i \chi t(l+2 m)^{2}-|\alpha|^{2} / 2},
\end{aligned}
$$

and when $\chi=0$ the Husimi function reduces to an exponential

$$
\begin{aligned}
Q(\alpha)_{\chi=0}= & \frac{1}{\pi} \mid \exp \left(\gamma_{4}+z^{2} \gamma_{3}-\frac{|z|^{2}}{2}-\frac{|\alpha|^{2}}{2}\right. \\
& \left.+z \alpha^{*} e^{\gamma_{2}-i \Omega_{0} t}+\alpha^{* 2} \gamma_{1} e^{-i 2 \Omega_{0} t}\right)\left.\right|^{2} .
\end{aligned}
$$

In Fig. $\underline{4}$ we show the Husimi function for $z=4$ and Hamiltonian parameters $\kappa=0.8$ and $\chi=0.25$ at different times. At time $t=0$ the state is a coherent state and its distribution is a Gaussian as shown in Fig. 4(a). As time evolves the distribution is smeared in a sort of ring. At $t=\pi / 4$ the state is no longer localized as shown in Fig. 4(b). Later on at $t=\pi$ the distribution has evolved into a superposition of four well defined peaks with Gaussian shape. At $t=6 \pi$ the distribution is now composed of only two peaks, a catlike state. Notice the presence of squeezing. In Figs. $\underline{4(\mathrm{e})}$ and $\underline{4(\mathrm{f})}$ we show the Husimi function at two nearby times $t=6.5 \pi$ and $t=6.55 \pi$. Notice that the distribution is localized in a
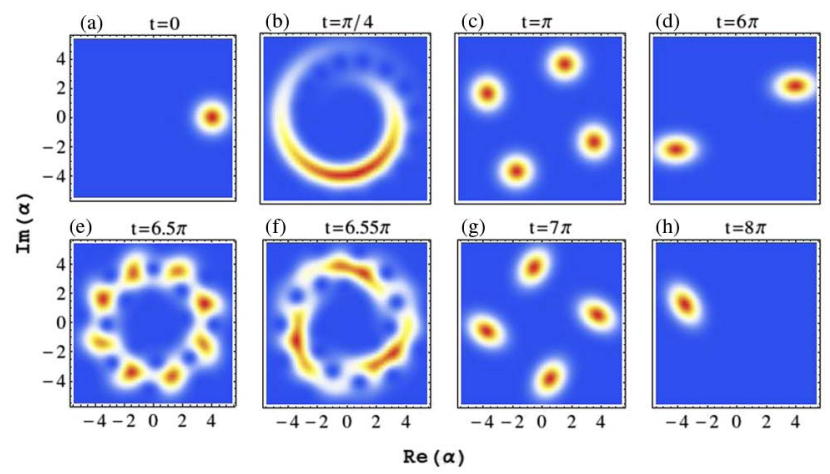

Fig. 4. Husimi $Q^{|z ; t\rangle\langle z ; t|}(\alpha)$ function with $\alpha=x+i y, z=4$, $\alpha_{0}=4$, and Hamiltonian parameters $\kappa=0.8, \chi=0.25$ at several times, indicated on top of each figure.

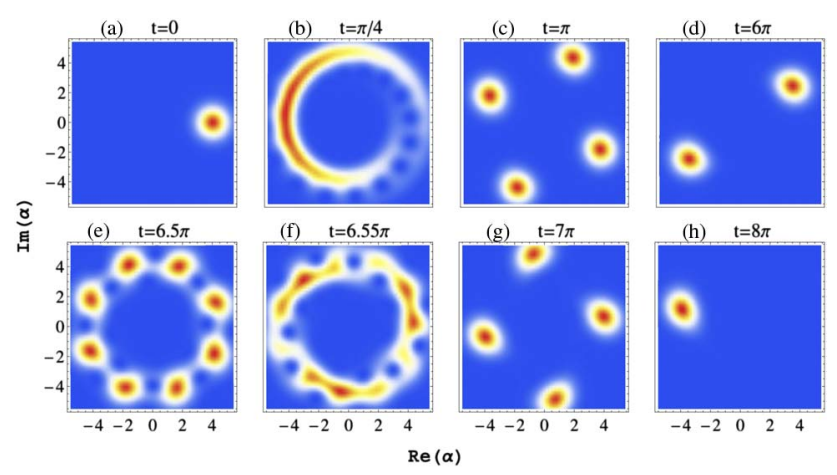

Fig. 5. Husimi $Q^{|z ; t\rangle\langle z ; t|}(\alpha)$ function with $\alpha=x+i y, z=4$, $\alpha_{0}=4$, and Hamiltonian parameters $\kappa=0.8, \chi=0.25$ at several times, indicated on top of each figure.

kind of ring whose radius is $\simeq \sqrt{18}$. At $t=7 \pi$ the distribution resembles that of $t=\pi$ and we can see that the states are squeezed. Finally at the revival time $T_{\text {rev }}=8 \pi$ the distribution is composed of a single peak that is not located at the same phase-space point it had at $t=0$. This is due to the temporal dependence of the frequency. Similar results have been shown in $[26]$.

Finally, in Fig. 5 we show the Husimi function obtained from the converged numerical results when the Schrödinger equation is solved with the Hamiltonian given by Eq. (). Here we present results for the same set of parameters and specific times as those used in Fig. 4. Notice the very close similarity between both figures for all times. This is an indication of the quality of our approximate interaction-picture Hamiltonian.

\section{CONCLUSIONS}

In this work we have considered the evolution of coherent states in a parametric oscillator immersed in a Kerr-like medium. Transforming the Hamiltonian into the interaction picture we obtained a time-dependent Hamiltonian that contains the number operator in an exponential. In order to have a more manageable Hamiltonian we approximate the exponential by 
its average value taken between a time-independent coherent state. With this simplification we can write the Hamiltonian in the interaction picture as an element of a finite Lie algebra whose time-evolution operator can be expressed as a product of exponentials. To show the quality of our methodology we calculated probability distributions, the auto-correlation function, and the Husimi distribution function for a case where neither $\kappa$ nor $\chi$ are negligible, that is, for a nonlinear parametric oscillator. We found that the approximation made in order to make the interaction-picture Hamiltonian a member of a finite Lie algebra is consistent with the converged numerical results.

Funding. Consejo Nacional de Ciencia y Tecnología (CONACyT) (166961); DGAPA-UNAM (IN108413).

Acknowledgment. We thank Reyes García for the maintenance of our computers. One of us (M. B.) would like to thank the Instituto de Ciencias Físicas for its hospitality.

\section{REFERENCES}

1. E. Schrödinger, "Der stetige Übergang von der mikro- zur makromechanik," Naturwissenschaften 14, 664-666 (1926).

2. R. J. Glauber, "Photon correlations," Phys. Rev. Lett. 10, 84-86 (1963).

3. R. J. Glauber, "Coherent and incoherent states of the radiation field," Phys. Rev. 131, 2766-2788 (1963).

4. J. R. Klauder, "The action option and a Feynman quantization of spinor fields in terms of ordinary c-numbers," Ann. Phys. 11, 123168 (1960)

5. J. Récamier, P. García de León, R. Jáuregui, A. Frank, and O. Castaños, "Coherent states for anharmonic diatomic molecules," Int. J. Quantum Chem. 89, 494-502 (2002).

6. M. M. Nieto and L. M. Simmons, "Coherent states for general potentials," Phys. Rev. Lett. 41, 207-210 (1978).

7. M. M. Nieto and L. M. Simmons, "Coherent states for general potentials. I. Formalism," Phys. Rev. D 20, 1321-1331 (1979).

8. M. M. Nieto and L. M. Simmons, "Coherent states for general potentials. III. Nonconfining one-dimensional examples," Phys. Rev. D 20, 1342-1350 (1979)

9. J. P. Gazeau and J. R. Klauder, "Coherent states for systems with discrete and continuous spectrum," J. Phys. A 32, 123-132 (1999).

10. V. I. Man'ko, G. Marmo, E. C. G. Sudarshan, and F. Zaccaria, "f-Oscillators and nonlinear coherent states," Phys. Scripta 55, 528-541 (1997).

11. B. Roy and P. Roy, "Time dependent nonclassical properties of even and odd nonlinear coherent states," Phys. Lett. A 263, 48-52 (1999).

12. J. Récamier and R. Jáuregui, "Construction of even and odd combinations of Morse-like coherent states," J. Opt. B 5, S365-S370 (2003).

13. B. Yurke and D. Stoler, "Generating quantum mechanical superpositions of macroscopically distinguishable states via amplitude dispersion," Phys. Rev. Lett. 57, 13-16 (1986).
14. S. Haroche, Exploring the Quantum Atoms, Cavities and Photons (Oxford, 2006).

15. H. R. Lewis, "Classical and quantum systems with time-dependent harmonic-oscillator-type Hamiltonians," Phys. Rev. Lett. 18, 510-512 (1967).

16. H. R. Lewis, "Class of exact invariants for classical and quantum timedependent harmonic oscillators," J. Math. Phys. 9, 1976-1986 (1968).

17. H. R. Lewis and W. B. Riesenfeld, "An exact quantum theory of the time-dependent harmonic oscillator and of a charged particle in a time-dependent electromagnetic field," J. Math. Phys. 10, 14581473 (1969).

18. V. Dodonov and O. Man'ko, "Universal invariants in quantum mechanics and physics of optical and particle beams," J. Russ. Laser Res. 21, 438-464 (2000).

19. V. V. Dodonov, Theory of Nonclassical States of Light (Taylor, 2003).

20. O. Castaños, D. Schuch, and O. Rosas-Ortiz, "Generalized coherent states for time-dependent and nonlinear Hamiltonian operators via complex Riccati equations," J. Phys. A 46, 075304 (2013).

21. B. Gazdy and D. A. Micha, "The linearly driven parametric oscillator: application to collisional energy transfer," J. Chem. Phys. 82, 49264936 (1985).

22. J. Récamier and R. Jáuregui, "Time-evolution operator for a forced parametric oscillator," Int. J. Quantum Chem. 62, 125-135 (1997).

23. T. Kiss, J. Janszky, and P. Adam, "Time evolution of harmonic oscillators with time-dependent parameters: a step-function approximation," Phys. Rev. A 49, 4935-4942 (1994).

24. V. V. Dodonov, A. B. Klimov, and D. E. Nikonov, "Quantum phenomena in nonstationary media," Phys. Rev. A 47, 4422-4429 (1993).

25. D. J. Wineland, C. Monroe, W. Itano, D. Leibfried, B. King, and D. Meekhof, "Experimental issues in coherent quantum-state manipulation of trapped atomic ions," arXiv:quant-ph/9710025 (1997).

26. R. de. J. León-Montiel and H. Moya-Cessa, "Generation of squeezed Schrödinger cats in a tunable cavity filled with a Kerr medium," J. Opt. 17, 065202 (2015).

27. D. F. Walls, Quantum Optics (Springer, 2008).

28. R. Boyd, Nonlinear Optics (Academic, 2008).

29. V. V. Dodonov, M. A. Marchiolli, Y. A. Korennoy, V. I. Man'ko, and Y. A. Moukhin, "Dynamical squeezing of photon-added coherent states," Phys. Rev. A 58, 4087-4094 (1998).

30. M. Berrondo and J. Récamier, "Dipole induced transitions in an anharmonic oscillator: a dynamical mean field model," Chem. Phys. Lett. 503, 180-184 (2011).

31. C. C. Gerry, Introductory Quantum Optics (Cambridge, 2005).

32. J. Wei and E. Norman, "Lie algebraic solution of linear differential equations," J. Math. Phys. 4, 575-581 (1963).

33. J. Wei and E. Norman, "On global representations of the solutions of linear differential equations as a product of exponentials," Proc. Am. Math. Soc. 15, 327-334 (1964).

34. R. Robinett, "Quantum wave packet revivals," Phys. Rep. 392, 1-119 (2004).

35. J. A. Yeazell and C. R. Stroud, "Observation of fractional revivals in the evolution of a Rydberg atomic wave packet," Phys. Rev. A 43, 5153-5156 (1991).

36. M. J. J. Vrakking, D. M. Villeneuve, and A. Stolow, "Observation of fractional revivals of a molecular wave packet," Phys. Rev. A 54, R37-R40 (1996). 